\title{
EXISTENCE OF POSITIVE SOLUTIONS FOR NONLINEAR ROBIN PROBLEMS WITH GRADIENT DEPENDENCE
}

\author{
Nikolaos S. Papageorgiou and Chao Zhang* \\ National Technical University, Department of Mathematics \\ Zografou Campus, Athens 15780, Greece; npapg@math.ntua.gr
}

Harbin Institute of Technology, Department of Mathematics and Institute for Advanced Study in Mathematics, Harbin 150001, P. R. China; czhangmath@hit.edu.cn

\begin{abstract}
We consider a nonlinear Robin problem driven by the $p$-Laplace differential operator and with a reaction term which depends also on the gradient (convection). Using a topological approach based on the Leray-Schauder alternative principle, we show that the problem has a positive smooth solution.
\end{abstract}

\section{Introduction}

Let $\Omega \subseteq \mathbf{R}^{N}$ be a bounded domain with a $C^{2}$-boundary $\partial \Omega$. In this paper we study the following nonlinear Robin problem with gradient dependence (convection):

$$
\begin{cases}-\Delta_{p} u(z)+\xi(z)|u(z)|^{p-2} u(z)=f(z, u(z), D u(z)) & \text { in } \Omega, \\ \frac{\partial u}{\partial n_{p}}+\beta(z)|u|^{p-2} u=0 & \text { on } \partial \Omega .\end{cases}
$$

In this problem $\Delta_{p}$ denotes the $p$-Laplace differential operator defined by

$$
\Delta_{p} u=\operatorname{div}\left(|D u|^{p-2} D u\right) \quad \text { for all } u \in W^{1, p}(\Omega), 1<p<\infty .
$$

The reaction term $f(z, x, y)$ is a Caratheodory perturbation (that is, for all $(x, y) \in \mathbf{R} \times \mathbf{R}^{N} z \rightarrow f(z, x, y)$ is measurable and for a.a. $z \in \Omega(x, y) \rightarrow f(z, x, y)$ is continuous). The dependence of $f$ on the gradient of the unknown function $u$, makes the problem nonvariational. In the boundary condition $\frac{\partial u}{\partial n_{p}}$ denotes the conormal derivative of $u$ for the $p$-Laplace differential operator. It is defined by extension of the map

$$
C^{1}(\bar{\Omega}) \ni u \longrightarrow|D u|^{p-2}(D u, n)_{\mathbf{R}^{N}}=|D u|^{p-2} \frac{\partial u}{\partial n} .
$$

Here $n(\cdot)$ denotes the outward unit normal on $\partial \Omega$.

The nonvariational character of (1.1), requires a topological approach based on the fixed point theory. Assuming that $f(z, \cdot, y)$ is $(p-1)$-sublinear near $+\infty$, we show that there exists a positive smooth solution for problem (1.1).

In the past, positive solutions for elliptic problems with convection were proved primarily for Dirichlet problems. In this direction we mention the works of de Figueiredo-Girardi-Matzeu [6], Girardi-Matzeu [12] (semilinear problems) and Bai

https://doi.org/10.5186/aasfm.2019.4437

2010 Mathematics Subject Classification: Primary 35J92, 35P30.

Key words: Convection, Leray-Schauder alternative principle, minimal positive solution, nonlinear regularity, nonlinear maximum principle.

*Corresponding author. 
[2], Faraci-Motreanu-Puglisi [4], Faria-Miyagaki-Motreanu-Tanaka [5], García Melián-Sabina de Lis-Takáč [7], Huy-Quan-Khanh [14], Ruiz [26], Tanaka [27] (nonlinear problems) together with Motreanu-Vetro-Vetro $[16,17]$ (nonlinear system problems). For Neumann and Robin problems, there are the recent works of GasinskiPapageorgiou [10], Papageorgiou-Radulescu-Repovs [24] (semilinear Neumann problems with a differential operator of the form $\left.\operatorname{div}(a(u) D u), u \in W^{1, p}(\Omega)\right)$ and BaiGasinski-Papageorgiou [3], Papageorgiou-Radulescu-Repovs [25] (nonlinear Robin problems). In these papers the hypotheses on the reaction are different and in many respects more restrictive.

\section{Preliminaries and hypotheses}

As we already mentioned our approach is topological and employs the LeraySchauder Alternative Principle (see Gasinski-Papageorgiou [8], p. 890). So, let $X, Y$ be two Banach spaces, $D \subseteq X$ nonempty and $f: D \rightarrow Y$. We say that $f(\cdot)$ is "compact", if it is continuous and maps bounded subsets of $D$ to relatively compact sets in $Y$. The Leray-Schauder Alternative Principle reads as follows:

Theorem 2.1. If $X$ is a Banach space, $C \subseteq X$ is nonempty, convex with $0 \in C$, $f: C \rightarrow C$ is compact and $E(f)=\{u \in C: u=\lambda f(u)$ for some $\lambda \in(0,1)\}$, then either $E(f)$ is unbounded or $f$ admits a fixed point.

The following spaces are important in the analysis of problem (1.1)

$$
W^{1, p}(\Omega), C^{1}(\bar{\Omega}) \text { and } L^{p}(\partial \Omega) .
$$

By $\|\cdot\|$ we denote the norm of the Sobolev space $W^{1, p}(\Omega)$. It is defined by

$$
\|u\|=\left[\|u\|_{p}^{p}+\|D u\|_{p}^{p}\right]^{1 / p} \quad \text { for all } u \in W^{1, p}(\Omega) .
$$

The space $C^{1}(\bar{\Omega})$ is an ordered Banach space with positive (order) cone given by

$$
C_{+}=\left\{u \in C^{1}(\bar{\Omega}): u(z) \geq 0 \text { for all } z \in \bar{\Omega}\right\} .
$$

This cone has a nonempty interior given by

$$
D_{+}=\left\{u \in C_{+}: u(z)>0 \text { for all } z \in \bar{\Omega}\right\} .
$$

Note that $D_{+}$is also the interior of $C_{+}$when this space is equipped with the weaker $C(\bar{\Omega})$-norm topology.

On $\partial \Omega$ we consider the $(N-1)$-dimensional Hausdorff (surface) measure $\sigma(\cdot)$. Using this measure on $\partial \Omega$, we can define in the usual way the boundary Lebesgue spaces $L^{q}(\partial \Omega)(1 \leq q \leq \infty)$. From the theory of Sobolev spaces, we know that there exists a unique continuous linear map $\gamma_{0}: W^{1, p}(\Omega) \rightarrow L^{p}(\partial \Omega)$, which is known as the "trace map", such that

$$
\gamma_{0}(u)=\left.u\right|_{\partial \Omega} \text { for all } u \in W^{1, p}(\Omega) \cap C(\bar{\Omega}) .
$$

So, the trace map extends the notion of boundary values to all Sobolev functions. The trace map is compact into $L^{q}(\partial \Omega)$ for all $q \in\left[1, \frac{(N-1) p}{N-p}\right)$ if $p<N$ and into $L^{q}(\partial \Omega)$ for all $1 \leq q<\infty$ if $N \leq p$. Moreover, we have

$$
\operatorname{im} \gamma_{0}=W^{\frac{1}{p^{\prime}}, p}(\partial \Omega) \quad \text { and } \quad \operatorname{ker} \gamma_{0}=W_{0}^{1, p}(\Omega) \quad\left(\frac{1}{p}+\frac{1}{p^{\prime}}=1\right) .
$$

In what follows, for the sake of notational simplicity, we drop the use of the trace map $\gamma_{0}(\cdot)$. All restrictions of Sobolev functions on $\partial \Omega$ are understood in the sense of traces. 
We introduce the hypotheses on the potential function $\xi(\cdot)$ and on the boundary coefficient $\beta(\cdot)$.

$H(\xi): \xi \in L^{\infty}(\Omega), \xi(z) \geq 0$ for a.a. $z \in \Omega$.

$H(\beta): \beta \in C^{0, \alpha}(\partial \Omega)$ with $\alpha \in(0,1), \beta(z) \geq 0$ for all $z \in \partial \Omega$.

$H_{0}: \xi \neq 0$ or $\beta \neq 0$.

Remark 2.2. When $\beta \equiv 0$, we recover the usual Neumann problem.

From Mugnai-Papageorgiou [18] (Lemma 4.11), we have:

Lemma 2.3. If $\xi \in L^{\infty}(\Omega), \xi(z) \geq 0$ for a.a. $z \in \Omega$ and $\xi \not \equiv 0$, then there exists $c_{1}>0$ such that

$$
\|D u\|_{p}^{p}+\int_{\Omega} \xi(z)|u|^{p} d z \geq c_{1}\|u\|^{p} \quad \text { for all } u \in W^{1, p}(\Omega) .
$$

Also, from Gasinski-Papageorgiou [11] (Proposition 2.4), we have:

Lemma 2.4. If $\beta \in L^{\infty}(\partial \Omega), \beta(z) \geq 0$ for $\sigma$-a.a. $z \in \partial \Omega, \beta \not \equiv 0$, then the map

$$
u \rightarrow|u|=\left[\|D u\|_{p}^{p}+\int_{\partial \Omega} \beta(z)|u|^{p} d \sigma\right]^{1 / p}
$$

defines an equivalent norm on $W^{1, p}(\Omega)$.

In the sequel we assume that hypotheses $\mathrm{H}(\xi), \mathrm{H}(\beta), \mathrm{H}_{0}$ hold. Let $\gamma: W^{1, p}(\Omega) \rightarrow$ $\mathbf{R}$ be the $C^{1}$-functional defined by

$$
\gamma(u)=\|D u\|_{p}^{p}+\int_{\Omega} \xi(z)|u|^{p} d z+\int_{\partial \Omega} \beta(z)|u|^{p} d \sigma \quad \text { for all } u \in W^{1, p}(\Omega) .
$$

On account of Lemmata 2.3 and 2.4, we see that we can find $c_{2}>0$ such that

$$
\gamma(u) \geq c_{2}\|u\|^{p} \quad \text { for all } u \in W^{1, p}(\Omega) .
$$

We consider the following nonlinear eigenvalue problem:

$$
\begin{cases}-\Delta_{p} u(z)+\xi(z)|u(z)|^{p-2} u(z)=\hat{\lambda}|u(z)|^{p-2} u(z) & \text { in } \Omega \\ \frac{\partial u}{\partial n_{p}}+\beta(z)|u|^{p-2} u=0 & \text { on } \partial \Omega .\end{cases}
$$

We say that $\hat{\lambda} \in \mathbf{R}$ is an "eigenvalue" of (2.2), if the problem admits a nontrivial solution $\hat{u} \in W^{1, p}(\Omega)$, known as an "eigenfunction" corresponding to $\hat{\lambda}$. From the nonlinear regularity theory of Lieberman [15] (Theorem 2), we have that $\hat{u} \in C^{1}(\bar{\Omega})$. There is a smallest eigenvalue $\hat{\lambda}_{1}$ with the following properties:

(a) $\hat{\lambda}_{1}$ is isolated (that is, there exists $\varepsilon>0$ such that the open interval $\left(\hat{\lambda}_{1}, \hat{\lambda}_{1}+\varepsilon\right)$ does not contain eigenvalues of (2.2)).

(b) $\hat{\lambda}_{1}$ is simple (that is, if $\hat{u}, \hat{v}$ are eigenfunctions corresponding to $\hat{\lambda}_{1}$, then $\hat{u}=\eta \hat{v}$ for some $\eta \in \mathbf{R} \backslash\{0\})$.

(c)

$$
\hat{\lambda}_{1}=\inf \left[\frac{\gamma(u)}{\|u\|_{p}^{p}}: u \in W^{1, p}(\Omega), u \neq 0\right]>0 \quad(\text { see }(2.1)) .
$$

The infimum in (2.3) is realized on the corresponding one-dimensional eigenspace (see property (b)). From the above properties it follows easily that the elements of this eigenspace do not change sign and as we already said belong in $C^{1}(\bar{\Omega})$. Let $\hat{u}_{1}$ denote the positive, $L^{p}$-normalized (that is, $\left\|\hat{u}_{1}\right\|_{p}=1$ ) eigenfunction corresponding to $\hat{\lambda}_{1}$. We have $\hat{u}_{1} \in C_{+} \backslash\{0\}$ and by the nonlinear maximum principle (see, for 
example, Gasinski-Papageorgiou [8, p. 738]), we have that $\hat{u}_{1} \in D_{+}$. We mention that every eigenfunction $\hat{u}$ corresponding to an eigenvalue $\hat{\lambda} \neq \hat{\lambda}_{1}$ is nodal (that is, sign changing).

As a simple consequence of the above properties we have the following lemma (see Papageorgiou-Radulescu-Repovs [22, Lemma 14]).

Lemma 2.5. If $\theta \in L^{\infty}(\Omega), \theta(z) \leq \hat{\lambda}_{1}$ for a.a. $z \in \Omega, \theta \not \equiv \hat{\lambda}_{1}$, then there exists $c_{3}>0$ such that

$$
c_{3}\|u\|^{p} \leq \gamma(u)-\int_{\Omega} \theta(z)|u|^{p} d z \quad \text { for all } u \in W^{1, p}(\Omega) .
$$

Next we introduce the hypotheses on the reaction term $f(z, x, y)$.

$\mathrm{H}(\mathrm{f}): f: \Omega \times \mathbf{R} \times \mathbf{R}^{N}$ is a Caratheodory function such that $f(z, 0,0)=0$ for a.a. $z \in \Omega, f(z, x, y)>0$ for a.a. $z \in \Omega$, all $x>0$, all $y \in \mathbf{R}^{N}$ and

(i) $f(z, x, y) \leq a(z)\left[1+x^{p-1}\right]+\mu|y|^{p-1}$ for a.a. $z \in \Omega$, all $x \geq 0$, all $y \in \mathbf{R}^{N}$ with $a \in L^{\infty}(\Omega)$ and $0<\mu<\hat{\lambda}_{1}^{1 / p}$

(ii) there exists a function $\theta \in L^{\infty}(\Omega)$ such that if $\mu_{0}=1-\frac{\mu}{\hat{\lambda}_{1}^{1 / p}}>0$, then

$$
\theta(z) \leq \hat{\lambda}_{1} \mu_{0} \quad \text { for a.a. } z \in \Omega, \quad \theta \not \equiv \hat{\lambda}_{1} \mu_{0}
$$

and for every $\varepsilon>0$, there exists $M_{\varepsilon}>0$ such that

$$
f(z, x, y) \leq[\theta(z)+\varepsilon] x^{p-1}+\mu|y|^{p-1}
$$

for a.a. $z \in \Omega$, all $x \geq M_{\varepsilon}$ and all $y \in \mathbf{R}^{N}$;

(iii) for every $M>0$, there exists $\hat{\eta}_{M} \in L^{\infty}(\Omega)$ such that

$$
\begin{gathered}
\hat{\eta}_{M}(z) \geq \hat{\lambda}_{1} \quad \text { for a.a. } z \in \Omega, \quad \hat{\eta}_{M} \not \equiv \hat{\lambda}_{1}, \\
\liminf _{x \rightarrow 0^{+}} \frac{f(z, x, y)}{x^{p-1}} \geq \hat{\eta}_{M}(z) \text { uniformly for a.a. } z \in \Omega, \text { all }|y| \leq M ;
\end{gathered}
$$

(iv) for every $\rho>0$ and every $M>0$, there exists $\hat{\xi}_{\rho, M}>0$ such that for a.a. $z \in \Omega$ and all $|y| \leq M$, the function

$$
x \rightarrow f(z, x, y)+\hat{\xi}_{\rho, M} x^{p-1}
$$

is nondecreasing on $[0, \rho]$ and for a.a. $z \in \Omega$, all $y \in \mathbf{R}^{N}$, the quotient function

$$
x \rightarrow \frac{f(z, x, y)}{x^{p-1}}
$$

is nonincreasing on $(0,+\infty)$.

Remark 2.6. Since we are looking for positive solutions and the above hypotheses concern $x \geq 0$, without any loss of generality we may assume that $f(z, x, y)=0$ for a.a. $z \in \Omega$, all $x \leq 0$, all $y \in \mathbf{R}^{N}$. Hypothesis $\mathrm{H}(\mathrm{f})(\mathrm{ii})$ implies that

$$
\limsup _{x \rightarrow+\infty} \frac{f(z, x, y)}{x^{p-1}} \leq \theta(z)
$$

uniformly for a.a. $z \in \Omega$ and all $y \in \mathbf{R}^{N}$ in a bounded set. So, this hypothesis is in fact a nonuniform nonresonance condition for $f(z, \cdot, y)$ at $+\infty$. 
Example 2.7. The following functions satisfy hypotheses H(f). For the sake of simplicity, we drop the $z$-dependence

$$
\begin{aligned}
f_{1}(x, y)= & \left\{\begin{array}{ll}
\hat{\eta} x^{p-1}+x^{q-1}|y|^{p-1} & \text { if } 0 \leq x \leq 1 \\
\hat{\eta} x^{\tau-1}+|y|^{p-1} & \text { if } 1<x
\end{array} \quad \text { with } \hat{\eta}>\hat{\lambda}_{1}, 1<\tau, q<p,\right. \\
f_{2}(x, y)= & \left\{\begin{array}{ll}
\hat{\eta} x^{p-1}+\mu|y|^{p-1} & \text { if } 0 \leq x \leq 1 \\
\theta x^{p-1}+(\hat{\eta}-\theta)|x|^{q-1}+\mu|y|^{p-1} & \text { if } 1<x
\end{array} \text { with } 0<\mu<\hat{\lambda}_{1}^{1 / p},\right. \\
& \theta<\hat{\lambda}_{1} \mu_{0}, \hat{\lambda}_{1}<\hat{\eta} \text { and } 1<q<p .
\end{aligned}
$$

Now let $B \subseteq C^{1}(\bar{\Omega})$ be bounded and let $M=\sup _{v \in B}\|v\|_{C^{1}(\bar{\Omega})}$. On account of hypotheses $\mathrm{H}(\mathrm{f})(\mathrm{i})$, (iii) and since $\hat{\eta}_{M} \in L^{\infty}(\Omega)$, given $\varepsilon>0$ and $r \in\left(p, p^{*}\right)$ (recall $p^{*}=\left\{\begin{array}{ll}\frac{N p}{N-p} & \text { if } p<N \\ +\infty & \text { if } N \leq p\end{array}\right)$, we can find $c_{4}=c_{4}(\varepsilon, r)>0$ such that

$$
f_{v}(z, x)=f(z, x, D v(z)) \geq\left[\hat{\eta}_{M}(z)-\varepsilon\right] x^{p-1}-c_{4} x^{r-1}
$$

for a.a. $z \in \Omega$, all $x \geq 0$, all $v \in B$.

Motivated by the unilateral growth condition $(2.4)$ on $f_{v}(z, \cdot)$ for all $v \in B$, we consider the following auxiliary nonlinear Robin problem

$$
\begin{cases}-\Delta_{p} u(z)+\xi(z) u(z)^{p-1}=\left[\hat{\eta}_{M}(z)-\varepsilon\right] u(z)^{p-1}-c_{4} u(z)^{r-1} & \text { in } \Omega \\ \frac{\partial u}{\partial n_{p}}+\beta(z) u^{p-1}=0 & \text { on } \partial \Omega, \quad u>0\end{cases}
$$

Proposition 2.8. If hypotheses $\mathrm{H}(\xi), \mathrm{H}(\beta), \mathrm{H}_{0}$ hold, then for all $\varepsilon>0$ small problem (2.5) admits a unique positive solution $\tilde{u} \in D_{+}$.

Proof. We consider the $C^{1}$-functional $\psi: W^{1, p}(\Omega) \rightarrow \mathbf{R}$ defined by

$$
\psi(u)=\frac{1}{p} \gamma(u)+\frac{c_{4}}{r}\left\|u^{+}\right\|_{r}^{r}-\frac{1}{p} \int_{\Omega}\left[\hat{\eta}_{M}(z)-\varepsilon\right]\left(u^{+}\right)^{p} d z \quad \text { for all } u \in W^{1, p}(\Omega) .
$$

Since $r>p$, we see that

$$
\psi(\cdot) \text { is coercive. }
$$

Also using the Sobolev embedding theorem and the compactness of the trace map, we have that

$$
\psi(\cdot) \text { is sequentially weakly lower semicontinuous. }
$$

So, by the Weierstrass-Tonelli theorem, we can find $\tilde{u} \in W^{1, p}(\Omega)$ such that

$$
\psi(\tilde{u})=\inf \left[\psi(u): u \in W^{1, p}(\Omega)\right]
$$

For $t>0$, we have

$$
\begin{aligned}
\psi\left(t \hat{u}_{1}\right) & =\frac{t^{p}}{p} \hat{\lambda}_{1}+\frac{t^{r}}{r} c_{4}\left\|\hat{u}_{1}\right\|_{r}^{r}-\frac{t^{p}}{p} \int_{\Omega} \hat{\eta}_{M}(z) \hat{u}_{1}^{p}+\frac{\varepsilon t^{p}}{p} \quad\left(\text { recall that }\left\|\hat{u}_{1}\right\|_{p}=1\right) \\
& \leq \frac{t^{p}}{p}\left[\int_{\Omega}\left(\hat{\lambda}_{1}-\hat{\eta}_{M}(z)\right) \hat{u}_{1}^{p} d z+\varepsilon\right]+\frac{t^{r}}{r} c_{4}\left\|\hat{u}_{1}\right\|_{r}^{r} .
\end{aligned}
$$

From the hypothesis on $\hat{\eta}_{M}(\cdot)$ (see $\left.\mathrm{H}(\mathrm{f})(\mathrm{iii})\right)$ and since $\hat{u}_{1} \in D_{+}$, we have

$$
c_{M}=\int_{\Omega}\left(\hat{\eta}_{M}(z)-\hat{\lambda}_{1}\right) \hat{u}_{1}^{p} d z>0
$$

So, we have

$$
\psi\left(t \hat{u}_{1}\right) \leq \frac{t^{p}}{p}\left(-c_{M}+\varepsilon\right)+\frac{t^{r}}{r} c_{4}\left\|\hat{u}_{1}\right\|_{r}^{r} .
$$


Choosing $\varepsilon \in\left(0, c_{M}\right)$ it follows that

$$
\psi\left(t \hat{u}_{1}\right) \leq-\frac{t^{p}}{p} c_{5}+\frac{t^{r}}{r} c_{4}\left\|\hat{u}_{1}\right\|_{r}^{r} \text { for some } c_{5}>0 \text {, all } t>0 .
$$

Since $r>p$, choosing $t \in(0,1)$ small, we conclude that

$$
\begin{aligned}
& \psi\left(t \hat{u}_{1}\right)<0, \\
\Longrightarrow & \psi(\tilde{u})<0=\psi(0) \quad(\text { see }(2.6)), \\
\Longrightarrow & \tilde{u} \neq 0 .
\end{aligned}
$$

From (2.6) we have

$$
\begin{aligned}
& \psi^{\prime}(\tilde{u})=0 \\
\Longrightarrow & \langle A(\tilde{u}), h\rangle+\int_{\Omega} \xi(z)|\tilde{u}|^{p-2} \tilde{u} h d z+\int_{\partial \Omega} \beta(z)|\tilde{u}|^{p-2} \tilde{u} h d \sigma \\
= & \int_{\Omega}\left(\hat{\eta}_{M}(z)-\varepsilon\right)\left(\tilde{u}^{+}\right)^{p-1} h d z-c_{4} \int_{\Omega}\left(\tilde{u}^{+}\right)^{r-1} h d z \quad \text { for all } h \in W^{1, p}(\Omega) .
\end{aligned}
$$

In $(2.7)$ we choose $h=-\tilde{u}^{-} \in W^{1, p}(\Omega)$. Then

$$
\begin{aligned}
& \gamma\left(\tilde{u}^{-}\right)=0, \\
\Longrightarrow & \hat{\lambda}_{1}\left\|\tilde{u}^{-}\right\|_{p}^{p} \leq 0 \quad(\text { see }(2.3)), \\
\Longrightarrow & \tilde{u} \geq 0, \tilde{u} \neq 0 .
\end{aligned}
$$

Then from (2.7) it follows that

$$
\begin{cases}-\Delta_{p} \tilde{u}(z)+\xi(z) \tilde{u}(z)^{p-1}=\left[\hat{\eta}_{M}(z)-\varepsilon\right] \tilde{u}(z)^{p-1}-c_{4} \tilde{u}(z)^{r-1} & \text { for a.a. } z \in \Omega \\ \frac{\partial \tilde{u}}{\partial n_{p}}+\beta(z) \tilde{u}^{p-1}=0 & \text { on } \partial \Omega\end{cases}
$$

(see Papageorgiou-Radulescu [19]).

From (2.8) and Proposition 2.10 of Papageorgiou-Radulescu [20], we have

$$
\tilde{u} \in L^{\infty}(\Omega) .
$$

Invoking Theorem 2 of Lieberman [15], we have

$$
\tilde{u} \in C_{+} \backslash\{0\} .
$$

From (2.5), hypothesis $H(\xi)$ and by taking $\varepsilon>0$ even smaller if necessary (so that $\left.\varepsilon<\hat{\lambda}_{1}\right)$, we have

$$
\begin{aligned}
& \Delta_{p} \tilde{u}(z) \leq\left[\|\xi\|_{\infty}+c_{4}\|\tilde{u}\|_{\infty}^{r-p}\right] \tilde{u}(z)^{p-1} \quad \text { for a.a. } z \in \Omega, \\
\Longrightarrow & \tilde{u} \in D_{+}
\end{aligned}
$$

(by the nonlinear maximum principle, see [8, p. 738]).

We show that this positive solution is unique. Indeed, suppose that $\tilde{v} \in W^{1, p}(\Omega)$ is another positive solution of (2.5). Again we have $\tilde{v} \in D_{+}$. Let $t>0$ be the biggest positive real such that

$$
t \tilde{v} \leq \tilde{u}
$$

Suppose that $t<1$. If $\rho=\|\tilde{u}\|_{\infty}$, then we can find $\tilde{\xi}_{\rho}>0$ such that for a.a. $z \in \Omega$, the function

$$
x \rightarrow\left[\hat{\eta}_{M}(z)-\varepsilon\right] x^{p-1}-c_{4} x^{r-1}+\tilde{\xi}_{\rho} x^{p-1}
$$


in nondecreasing on $[0, \rho]$. We have

$$
\begin{aligned}
& -\Delta_{p}(t \tilde{v})+\left[\xi(z)+\tilde{\xi}_{\rho}\right](t \tilde{v})^{p-1} \\
& =t^{p-1}\left[-\Delta_{p} \tilde{v}+\left(\xi(z)+\tilde{\xi}_{\rho}\right) \tilde{v}^{p-1}\right] \\
& =t^{p-1}\left[\left(\hat{\eta}_{M}(z)-\varepsilon\right) \tilde{v}^{p-1}-c_{4} \tilde{v}^{r-1}+\tilde{\xi}_{\rho} \tilde{v}^{p-1}\right] \\
& \leq\left[\hat{\eta}_{M}(z)-\varepsilon\right](t \tilde{v})^{p-1}-c_{4}(t \tilde{v})^{r-1}+\tilde{\xi}_{\rho}(t \tilde{v})^{p-1} \quad(\text { since } t<1 \text { and } r>p) \\
& \leq\left[\hat{\eta}_{M}(z)-\varepsilon\right] \tilde{u}^{p-1}-c_{4} \tilde{u}^{r-1}+\tilde{\xi}_{\rho} \tilde{u}^{p-1} \quad(\text { see }(2.9)) \\
& =-\Delta_{p} \tilde{u}+\left[\xi(z)+\tilde{\xi}_{\rho}\right] \tilde{u}^{p-1} \quad \text { for a.a. } z \in \Omega .
\end{aligned}
$$

Note that since $\tilde{v} \in D_{+}$, we have $\widetilde{m}=\min _{\bar{\Omega}} \tilde{v}>0$. Then

$$
c_{4}\left[t^{p-1}-t^{r-1}\right] \tilde{v}(z) \geq c_{4}\left[t^{p-1}-t^{r-1}\right] \widetilde{m}>0 \quad(\text { since } t<1 \text { and } r>p) .
$$

Then from (2.10) and Proposition 2.10 of Papageorgiou-Radulescu-Repovs [23] it follows that

$$
\tilde{u}-t \tilde{v} \in \operatorname{int} C_{+},
$$

which contradicts the maximality of $t>0$. Hence $t \geq 1$ and so

$$
\tilde{v} \leq \tilde{u} \quad(\operatorname{see}(2.9))
$$

In the above argument we reverse the roles of $\tilde{u}$ and $\tilde{v}$ and obtain

$$
\begin{aligned}
\tilde{u} & \leq \tilde{v}, \\
\Longrightarrow \tilde{u} & =\tilde{v} .
\end{aligned}
$$

This proves the uniqueness of the positive solution of problem (2.5).

\section{Positive solution}

We employ the "freezing method". Namely, we fix (freeze) the gradient term in the reaction $f(z, x, y)$ and study the resulting problem which has variational structure. We solve this problem and we show that it has a positive solution. Moreover, we show that it has a smallest positive solution (minimal positive solution). So, we have a canonical way to choose a solution of the "frozen problem". We show that this minimal solution map has a fixed point. This is the desired positive solution of (1.1).

We start implementing this approach. We fix $v \in C^{1}(\bar{\Omega})$ and consider the following nonlinear Robin problem

$$
\begin{cases}-\Delta_{p} u(z)+\xi(z) u(z)^{p-1}=f(z, u(z), D v(z)) & \text { in } \Omega, \\ \frac{\partial u}{\partial n_{p}}+\beta(z) u^{p-1}=0 & \text { on } \partial \Omega, \quad u>0 .\end{cases}
$$

Proposition 3.1. If hypotheses $\mathrm{H}(\xi), \mathrm{H}(\beta), \mathrm{H}_{0}, \mathrm{H}(\mathrm{f})$ hold and $v \in C^{1}(\bar{\Omega})$, then problem (3.1) admits a positive solution $\hat{u}_{v} \in D_{+}$.

Proof. Consider the $C^{1}$-functional $\hat{\varphi}_{v}: W^{1, p}(\Omega) \rightarrow \mathbf{R}$ defined by

$$
\hat{\varphi}_{v}(u)=\frac{1}{p} \gamma(u)-\int_{\Omega} F_{v}\left(z, u^{+}(z)\right) d z \quad \text { for all } u \in W^{1, p}(\Omega),
$$

where $F_{v}(z, x)=\int_{0}^{x} f(z, x, D v(z)) d z$.

Hypotheses $\mathrm{H}(\mathrm{f})(\mathrm{i})$, (ii), imply that given $\varepsilon>0$, we can find $c_{6}>0$ such that

$$
F_{v}(z, x) \leq \frac{1}{p}[\theta(z)+\varepsilon] x^{p}+c_{6} \quad \text { for a.a. } z \in \Omega \text {, all } x \geq 0 \quad\left(\text { recall } v \in C^{1}(\bar{\Omega})\right) .
$$


We have

$$
\begin{aligned}
\hat{\varphi}_{v}(u) & \geq \frac{1}{p} \gamma(u)-\frac{1}{p} \int_{\Omega}[\theta(z)+\varepsilon]|u|^{p} d z-c_{7} \quad \text { for some } c_{7}>0 \\
& \geq \frac{1}{p}\left[c_{3}-\varepsilon\right]\|u\|^{p}-c_{7} \quad(\text { see Lemma 2.5). }
\end{aligned}
$$

Choosing $\varepsilon \in\left(0, c_{3}\right)$, we infer that

$$
\hat{\varphi}_{v}(\cdot) \text { is coercive. }
$$

Also, from the Sobolev embedding theorem and the compactness of the trace map, we have that

$$
\hat{\varphi}_{v}(\cdot) \text { is sequentially weakly lower semicontinuous. }
$$

So, we can find $\hat{u}_{v} \in W^{1, p}(\Omega)$ such that

$$
\hat{\varphi}_{v}\left(\hat{u}_{v}\right)=\inf \left[\hat{\varphi}_{v}(u): u \in W^{1, p}(\Omega)\right]
$$

Let $M=\|v\|_{C^{1}(\bar{\Omega})}$. Then on account of hypothesis $\mathrm{H}(\mathrm{f})($ iii), given $\varepsilon>0$ we can find $\delta>0$ such that

$$
F_{v}(z, x) \geq \frac{1}{p}\left[\hat{\eta}_{M}(z)-\varepsilon\right] x^{p} \quad \text { for a.a. } z \in \Omega, \text { all } 0 \leq x \leq \delta .
$$

Recall that $\hat{u}_{1} \in D_{+}$. So, we can find $t \in(0,1)$ small such that

$$
t \hat{u}_{1}(z) \in(0, \delta] \text { for all } z \in \bar{\Omega} \text {. }
$$

As in the proof of Proposition 2.8, using (3.4) and (3.5), we show that for $t \in(0,1)$ small we have

$$
\begin{aligned}
& \hat{\varphi}_{v}\left(t \hat{u}_{1}\right)<0 \\
\Longrightarrow & \hat{\varphi}_{v}\left(\hat{u}_{v}\right)<0=\hat{\varphi}_{v}(0) \quad(\text { see }(3.3)), \\
\Longrightarrow & \hat{u}_{v} \neq 0 .
\end{aligned}
$$

From (3.3), we have

$$
\begin{aligned}
& \hat{\varphi}_{v}^{\prime}\left(\hat{u}_{v}\right)=0 \\
\Longrightarrow & \left\langle A\left(\hat{u}_{v}\right), h\right\rangle+\int_{\Omega} \xi(z)\left|\hat{u}_{v}\right|^{p-2} \hat{u}_{v} h d z+\int_{\partial \Omega} \beta(z)\left|\hat{u}_{v}\right|^{p-2} \hat{u}_{v} h d \sigma \\
= & \int_{\Omega} f_{v}\left(z, \hat{u}_{v}^{+}\right) h d z \quad \text { for all } h \in W^{1, p}(\Omega) .
\end{aligned}
$$

In (3.6) we choose $h=-\hat{u}_{v}^{-} \in W^{1, p}(\Omega)$. Then

$$
\begin{aligned}
& \gamma\left(\hat{u}_{v}^{-}\right)=0, \\
\Longrightarrow & \hat{\lambda}_{1}\left\|\hat{u}_{v}^{-}\right\|_{p}^{p} \leq 0 \quad(\text { see }(2.3)), \\
\Longrightarrow & \hat{u}_{v} \geq 0, \quad \hat{u}_{v} \neq 0 .
\end{aligned}
$$

From (3.6) we have

$$
\begin{cases}-\Delta_{p} \hat{u}_{v}(z)+\xi(z) \hat{u}_{v}(z)^{p-1}=f\left(z, \hat{u}_{v}(z), D v(z)\right) & \text { for a.a. } z \in \Omega \\ \frac{\partial \hat{u}_{v}}{\partial n_{p}}+\beta(z) \hat{u}_{v}^{p-1}=0 & \text { on } \partial \Omega\end{cases}
$$

(see Papageorgiou-Radulescu [19]).

As before from (3.7) and the nonlinear regularity theory we infer that

$$
\hat{u}_{v} \in C_{+} \backslash\{0\} .
$$


From (3.7) and since $f\left(z, \hat{u}_{v}(z), D v(z)\right) \geq 0$ for a.a. $z \in \Omega$, we have

$$
\Delta_{p} \hat{u}_{v}(z) \leq\|\xi\|_{\infty} \hat{u}_{v}(z)^{p-1} \quad \text { for a.a. } z \in \Omega,
$$

$\Longrightarrow \hat{u}_{v} \in D_{+} \quad$ (see Gasinski-Papageorgiou [8, p. 738]).

We will show the existence of a minimal positive solution. Let $S_{v}^{+}$be the set of positive solutions of problem (3.1). We have just seen (see Proposition 3.1) that $\emptyset \neq S_{v}^{+} \subseteq D_{+}$. Moreover, from Papageorgiou-Radulescu-Repovs [21] (see the proof of Proposition 7), we know that $S_{v}^{+}$is downward directed (that is, if $u_{1}, u_{2} \in S_{v}^{+}$, then there exists $u \in S_{v}^{+}$such that $\left.u \leq u_{1}, u \leq u_{2}\right)$.

Proposition 3.2. If hypotheses $\mathrm{H}(\xi), \mathrm{H}(\beta), \mathrm{H}_{0}, \mathrm{H}(\mathrm{f})$ hold and $v \in C^{1}(\bar{\Omega})$, then there exists $u_{v}^{*} \in S_{v}^{+}$such that $u_{v}^{*} \leq u$ for all $u \in S_{v}^{+}$.

Proof. Invoking Lemma 3.10, p. 178, of Hu-Papageorgiou [13], we can find $\left\{u_{n}\right\}_{n \geq 1} \subseteq S_{v}^{+}$decreasing such that

$$
\inf S_{v}^{+}=\inf _{n \geq 1} u_{n} .
$$

We have

$$
\left\langle A\left(u_{n}\right), h\right\rangle+\int_{\Omega} \xi(z) u_{n}^{p-1} h d z+\int_{\partial \Omega} \beta(z) u_{n}^{p-1} h d \sigma=\int_{\Omega} f\left(z, u_{n}, D v\right) h d z
$$

for all $h \in W^{1, p}(\Omega)$, all $n \in \mathbf{N}$.

Since $0 \leq u_{n} \leq u_{1} \in D_{+}$for all $n \in \mathbf{N}$, from (3.8), hypothesis $\mathrm{H}(\mathrm{f})(\mathrm{i})$ and Lemmata 2.3 and 2.4 it follows that

$$
\left\{u_{n}\right\}_{n \geq 1} \subseteq W^{1, p}(\Omega) \text { is bounded. }
$$

So, we may assume that

$$
u_{n} \stackrel{w}{\longrightarrow} \hat{u}_{v}^{*} \text { in } W^{1, p}(\Omega) \text { and } u_{n} \rightarrow \hat{u}_{v}^{*} \text { in } L^{p}(\Omega) \text { and in } L^{p}(\partial \Omega) .
$$

In (3.8) we choose $h=u_{n}-\hat{u}_{v}^{*} \in W^{1, p}(\Omega)$ and pass to the limit as $n \rightarrow+\infty$. Then

$$
\begin{aligned}
& \lim _{n \rightarrow+\infty}\left\langle A\left(u_{n}\right), u_{n}-\hat{u}_{v}^{*}\right\rangle=0, \\
\Longrightarrow & u_{n} \rightarrow \hat{u}_{v}^{*} \quad \text { in } W^{1, p}(\Omega)
\end{aligned}
$$

(see Papageorgiou-Radulescu-Repovs [23, Proposition 2.7]).

So, if in (3.8) we pass to the limit as $n \rightarrow+\infty$ and use (3.10), then

$$
\left\langle A\left(\hat{u}_{v}^{*}\right), h\right\rangle+\int_{\Omega} \xi(z)\left(\hat{u}_{v}^{*}\right)^{p-1} h d z+\int_{\partial \Omega} \beta(z)\left(\hat{u}_{v}^{*}\right)^{p-1} h d \sigma=\int_{\Omega} f\left(z, \hat{u}_{v}^{*}, D v\right) h d z
$$

for all $h \in W^{1, p}(\Omega)$.

Therefore $\hat{u}_{v}^{*} \in C_{+}$is a solution of (3.1). We will show that $\hat{u}_{v}^{*} \neq 0$. To this end, let $\hat{u} \in S_{v}^{+}$and consider the Caratheodory function $k(z, x)$ defined by

$$
k(z, x)= \begin{cases}0 & \text { if } x<0 \\ {\left[\hat{\eta}_{M}(z)-\varepsilon\right] x^{p-1}-c_{4} x^{r-1}} & \text { if } 0 \leq x \leq \hat{u}(z) \\ {\left[\hat{\eta}_{M}(z)-\varepsilon\right] \hat{u}(z)^{p-1}-c_{4} \hat{u}(z)^{r-1}} & \text { if } \hat{u}(z)<x\end{cases}
$$

We set $K(z, x)=\int_{0}^{x} k(z, s) d s$ and consider the $C^{1}$-functional $\hat{\psi}: W^{1, p}(\Omega) \rightarrow \mathbf{R}$ defined by

$$
\hat{\psi}(u)=\frac{1}{p} \gamma(u)-\int_{\Omega} K(z, u) d z \quad \text { for all } u \in W^{1, p}(\Omega) .
$$


Clearly $\hat{\psi}(\cdot)$ is coercive (see (3.11)). Also, it is sequentially weakly lower semicontinuous. So, we can find $\bar{u} \in W^{1, p}(\Omega)$ such that

$$
\hat{\psi}(\bar{u})=\inf \left[\hat{\psi}(u): u \in W^{1, p}(\Omega)\right]
$$

As in the proof of Proposition 2.8, since $r>p$, we have

$$
\begin{aligned}
& \hat{\psi}(\bar{u})<0=\hat{\psi}(0), \\
\Longrightarrow & \bar{u} \neq 0 .
\end{aligned}
$$

From (3.12) we have

$$
\begin{aligned}
& \hat{\psi}^{\prime}(\bar{u})=0 \\
\Longrightarrow & \langle A(\bar{u}), h\rangle+\int_{\Omega} \xi(z)|\bar{u}|^{p-2} \bar{u} h d z+\int_{\partial \Omega} \beta(z)|\bar{u}|^{p-2} \bar{u} h d \sigma=\int_{\Omega} k(z, \bar{u}) h d z
\end{aligned}
$$

for all $h \in W^{1, p}(\Omega)$.

In (3.13) we choose $h=-\bar{u}^{-} \in W^{1, p}(\Omega)$. Then using (3.11) we obtain

$$
\begin{aligned}
& \gamma\left(\bar{u}^{-}\right)=0, \\
\Longrightarrow & \hat{\lambda}_{1}\left\|\bar{u}^{-}\right\|_{p}^{p} \leq 0 \quad(\text { see }(2.3)), \\
\Longrightarrow & \bar{u} \geq 0, \bar{u} \neq 0 .
\end{aligned}
$$

Next in (3.13) we choose $h=(\bar{u}-\hat{u})^{+} \in W^{1, p}(\Omega)$. Then

$$
\begin{aligned}
\langle & \left.A(\bar{u}),(\bar{u}-\hat{u})^{+}\right\rangle+\int_{\Omega} \xi(z) \bar{u}^{p-1}(\bar{u}-\hat{u})^{+} d z+\int_{\partial \Omega} \beta(z) \bar{u}^{p-1}(\bar{u}-\hat{u})^{+} d \sigma \\
= & \int_{\Omega}\left[\left(\hat{\eta}_{M}(z)-\varepsilon\right) \hat{u}^{p-1}-c_{4} \hat{u}^{r-1}\right](\bar{u}-\hat{u})^{+} d z \quad(\text { see }(3.11)) \\
\leq & \int_{\Omega} f(z, \hat{u}, D v)(\bar{u}-\hat{u})^{+} d z \quad(\operatorname{see}(2.4)) \\
= & \left\langle A(\hat{u}),(\bar{u}-\hat{u})^{+}\right\rangle+\int_{\Omega} \xi(z) \hat{u}^{p-1}(\bar{u}-\hat{u})^{+} d z \\
& +\int_{\partial \Omega} \beta(z) \hat{u}^{p-1}(\bar{u}-\hat{u})^{+} d \sigma \quad\left(\text { since } \hat{u} \in S_{v}^{+}\right), \\
\Longrightarrow & \bar{u} \leq \hat{u} .
\end{aligned}
$$

Therefore we have proved that

$$
\bar{u} \in[0, \hat{u}]=\left\{y \in W^{1, p}(\Omega): 0 \leq y(z) \leq \hat{u}(z) \text { for a.a. } z \in \Omega\right\}, \quad \bar{u} \neq 0 .
$$

From (3.13), (3.11) and (3.14), we infer that

$$
\begin{aligned}
& -\Delta_{p} \bar{u}(z)+\xi(z) \bar{u}(z)^{p-1}=\left[\hat{\eta}_{M}(z)-\varepsilon\right] \bar{u}(z)^{p-1}-c_{4} \bar{u}(z)^{r-1} \quad \text { for a.a. } z \in \Omega, \\
& \frac{\partial \bar{u}}{\partial n_{p}}+\beta(z) \bar{u}^{p-1}=0 \quad \text { on } \partial \Omega, \\
\Longrightarrow & \bar{u}=\tilde{u} \in D_{+} \quad(\text { see Proposition } 2.8), \\
\Longrightarrow & \tilde{u} \leq \hat{u} \quad \text { for all } \hat{u} \in S_{v}^{+} .
\end{aligned}
$$

Therefore we have

$$
\begin{aligned}
& \tilde{u} \leq u_{n} \quad \text { for all } n \in \mathbf{N}, \\
\Longrightarrow & \tilde{u} \leq \hat{u}_{v}^{*} \quad(\text { see }(3.10)), \\
\Longrightarrow & \hat{u}_{v}^{*} \neq 0 .
\end{aligned}
$$


We conclude that $u_{v}^{*} \in S_{v}^{+} \subseteq D_{+}$and $u_{v}^{*}=\inf S_{v}^{+}$.

So, we can define the minimal solution map $\tau: C_{+} \rightarrow C_{+}$by

$$
\tau(v)=\hat{u}_{v}^{*} .
$$

Proposition 3.3. If hypotheses $\mathrm{H}(\xi), \mathrm{H}(\beta), \mathrm{H}_{0}, \mathrm{H}(\mathrm{f})$ hold, then the minimal solution map $\tau: C_{+} \rightarrow C_{+}$is compact.

Proof. First we show that $\tau(\cdot)$ is continuous. So, let $v_{n} \rightarrow v$ in $C_{+} \subseteq C^{1}(\bar{\Omega})$ and let $\hat{u}_{n}^{*}=\tau\left(v_{n}\right)$ for all $n \in \mathbf{N}$. We have

$$
\begin{aligned}
& \left\langle A\left(\hat{u}_{n}^{*}\right), h\right\rangle+\int_{\Omega} \xi(z)\left(\hat{u}_{n}^{*}\right)^{p-1} h d z+\int_{\partial \Omega} \beta(z)\left(\hat{u}_{n}^{*}\right)^{p-1} h d \sigma \\
& =\int_{\Omega} f\left(z, \hat{u}_{n}^{*}, D v_{n}\right) h d z \quad \text { for all } h \in W^{1, p}(\Omega) .
\end{aligned}
$$

Let $\rho=\max _{n \geq 1}\left\|v_{n}\right\|_{C^{1}(\bar{\Omega})}$ and $B_{0}=\bar{B}_{\rho}(0)=\left\{y \in \mathbf{R}^{N}:|y| \leq \rho\right\}$. On account of hypotheses $\mathrm{H}(\mathrm{f})(\mathrm{i})$, (ii), given $\varepsilon>0$, we can find $c_{8}=c_{8}(\varepsilon)>0$ such that

$$
f(z, x, y) \leq[\theta(z)+\varepsilon] x^{p-1}+c_{8} \quad \text { for a.a. } z \in \Omega \text {, all } x \geq 0 \text {, all } y \in B_{0},
$$

In (3.15) we choose $h=\hat{u}_{n}^{*} \in W^{1, p}(\Omega)$. Then

$$
\gamma\left(\hat{u}_{n}^{*}\right)=\int_{\Omega} f\left(z, \hat{u}_{n}^{*}, D v_{n}\right) u_{n}^{*} d z \leq \int_{\Omega}[\theta(z)+\varepsilon]\left(\hat{u}_{n}^{*}\right)^{p} d z+c_{8} \int_{\Omega} \hat{u}_{n}^{*} d z
$$

$\Longrightarrow\left[c_{3}-\varepsilon\right]\left\|\hat{u}_{n}^{*}\right\|^{p-1} \leq c_{9} \quad$ for some $c_{9}>0$, all $n \in \mathbf{N} \quad$ (see Lemma 2.5),

$\Longrightarrow\left\{\hat{u}_{n}^{*}\right\}_{n \geq 1} \subseteq W^{1, p}(\Omega)$ is bounded (choose $\varepsilon \in\left(0, c_{3}\right)$ ).

Invoking Proposition 2.10 of Papageorgiou-Radulescu [20], we can find $c_{10}>0$ such that

$$
\left\|\hat{u}_{n}^{*}\right\|_{\infty} \leq c_{10} \text { for all } n \in \mathbf{N} .
$$

Then using Theorem 2 of Lieberman [15], we can find $s \in(0,1)$ and $c_{11}>0$ such that

$$
\hat{u}_{n}^{*} \in C^{1, s}(\bar{\Omega}), \quad\left\|\hat{u}_{n}^{*}\right\|_{C^{1, s}(\bar{\Omega})} \leq c_{11} \quad \text { for all } n \in \mathbf{N} .
$$

Exploiting the compact embedding of $C^{1, s}(\bar{\Omega})$ into $C^{1}(\bar{\Omega})$, at least for a subsequence we have

$$
\hat{u}_{n}^{*} \rightarrow \hat{u}^{*} \quad \text { in } C^{1}(\bar{\Omega}) \quad \text { and } \quad \hat{u}^{*} \in S_{v}^{+} \quad(\operatorname{see}(3.15)) .
$$

We will show that $\hat{u}^{*}=\tau(v)=\hat{u}_{v}^{*}$ and this will imply that the original sequence converges in $C^{1}(\bar{\Omega})$ to $\hat{u}^{*}=\tau(v)=\hat{u}_{v}^{*}$ and so we have the continuity of $\tau(\cdot)$.

Claim. We can find a sequence $\left\{\hat{u}_{n}\right\}_{n \geq 1}$ with $\hat{u}_{n} \in S_{v_{n}}^{+}$for all $n \in \mathbf{N}$ such that

$$
\hat{u}_{n} \rightarrow \tau(v)=u_{v}^{*} \quad \text { in } C^{1}(\bar{\Omega}) .
$$

To establish the Claim, we start by considering the following nonlinear Robin problem

$$
\begin{cases}-\Delta_{p} u(z)+\xi(z)|u(z)|^{p-2} u(z)=f\left(z, u_{v}^{*}(z), D v_{n}(z)\right) & \text { in } \Omega, \\ \frac{\partial u}{\partial n_{p}}+\beta(z)|u|^{p-2} u=0 & \text { on } \partial \Omega .\end{cases}
$$

This problem has a unique solution (note that the map $u \rightarrow A(u)+\xi(z)|u|^{p-2} u$ is continuous, strictly monotone (hence maximal monotone) and coercive (see Lemma 2.3). 
Let $u_{n}^{0} \in D_{+}(n \in \mathbf{N})$ be this unique solution. The nonlinear regularity theory of Lieberman [15] implies that there exist $\eta \in(0,1)$ and $c_{12}>0$ such that

$$
u_{n}^{0} \in C^{1, \eta}(\bar{\Omega}) \text { and }\left\|u_{n}^{0}\right\|_{C^{1, \eta}(\bar{\Omega})} \leq c_{12} \quad \text { for all } n \in \mathbf{N} .
$$

So, by passing to a subsequence if necessary, we have

$$
u_{n}^{0} \rightarrow \hat{u}^{0} \quad \text { in } C^{1}(\bar{\Omega}) \text { as } n \rightarrow+\infty .
$$

In the limit we have

$$
\begin{aligned}
& -\Delta_{p} \hat{u}^{0}(z)+\xi(z) \hat{u}^{0}(z)^{p-1}=f\left(z, u_{v}^{*}(z), D v(z)\right) \quad \text { for a.a. } z \in \Omega, \\
& \frac{\partial \hat{u}^{0}}{\partial n_{p}}+\beta(z)\left(\hat{u}^{0}\right)^{p-1}=0 \quad \text { on } \partial \Omega, \\
\Longrightarrow & \hat{u}^{0}=u_{v}^{*} .
\end{aligned}
$$

Hence for the original sequence we have

$$
u_{n}^{0} \rightarrow u_{v}^{*} \quad \text { in } C^{1}(\bar{\Omega}) .
$$

Next we consider the following nonlinear Robin problem

$$
\begin{cases}-\Delta_{p} u(z)+\xi(z)|u(z)|^{p-2} u(z)=f\left(z, u_{n}^{0}(z), D v_{n}(z)\right) & \text { in } \Omega \\ \frac{\partial u}{\partial n_{p}}+\beta(z)|u|^{p-2} u=0 & \text { on } \partial \Omega .\end{cases}
$$

As above this problem too has a unique solution $u_{n}^{1} \in D_{+}, n \in \mathbf{N}$ and

$$
u_{n}^{1} \rightarrow u_{v}^{*} \quad \text { in } C^{1}(\bar{\Omega}) \text { as } n \rightarrow+\infty .
$$

We continue this way and generate a sequence $\left\{u_{n}^{k}\right\}_{k, n \in \mathbf{N}}$ such that

$$
\begin{cases}-\Delta_{p} u_{n}^{k}(z)+\xi(z) u_{n}^{k}(z)^{p-1}=f\left(z, u_{n}^{k-1}(z), D v_{n}(z)\right) & \text { for a.a. } z \in \Omega, \\ \frac{\partial u_{n}^{k}}{\partial n_{p}}+\beta(z)\left(u_{n}^{k}\right)^{p-1}=0 & \text { on } \partial \Omega,\end{cases}
$$

and

$$
u_{n}^{k} \rightarrow u_{v}^{*} \quad \text { in } C^{1}(\bar{\Omega}) \text { as } n \rightarrow+\infty \text { for all } k \in \mathbf{N} .
$$

For every fixed $n \in \mathbf{N}$, from (3.20) and the nonlinear regularity theory (see [20, Proposition 2.10] and [15, Theorem 2]), we have that

$$
\left\{u_{n}^{k}\right\}_{k \in \mathbf{N}} \subseteq C^{1}(\bar{\Omega}) \text { is relatively compact. }
$$

So, we can find a subsequence $\left\{u_{n}^{k_{m}}\right\}_{m \in \mathbf{N}}$ of $\left\{u_{n}^{k}\right\}_{k \in \mathbf{N}}$ such that

$$
u_{n}^{k_{m}} \rightarrow \hat{u}_{n} \quad \text { in } C^{1}(\bar{\Omega}) \text { as } m \rightarrow+\infty .
$$

From (3.20) we obtain

$$
\begin{cases}-\Delta_{p} \hat{u}_{n}(z)+\xi(z) \hat{u}_{n}(z)^{p-1}=f\left(z, \hat{u}_{n}(z), D v_{n}(z)\right) & \text { for a.a. } z \in \Omega, \\ \frac{\partial \hat{u}_{n}}{\partial n_{p}}+\beta(z) \hat{u}_{n}^{p-1}=0 & \text { on } \partial \Omega, \quad n \in \mathbf{N} .\end{cases}
$$

From (3.23) we have that $\left\{\hat{u}_{n}\right\}_{n \geq 1} \subseteq C^{1}(\bar{\Omega})$ is relatively compact. So, from (3.21), (3.22) and the double limit lemma (see Aubin [1] and Gasinski-Papageorgiou [9, Problem 1.175, p. 61]), we have

$$
\hat{u}_{n} \in S_{v_{n}}^{+}, n \in \mathbf{N} \quad(\text { see }(3.23)) \quad \text { and } \quad \hat{u}_{n} \rightarrow u_{v}^{*} \quad \text { in } C^{1}(\bar{\Omega}) \text { as } n \rightarrow+\infty \text {. }
$$

This proves the Claim.

On account of the Claim, we can find $\hat{u}_{n} \in S_{v_{n}}^{+}, n \in \mathbf{N}$ such that

$$
\hat{u}_{n} \rightarrow u_{v}^{*}=\tau(v) \quad \text { in } C^{1}(\bar{\Omega}) .
$$


We have

$$
\begin{aligned}
& u_{n}^{*}=\tau\left(v_{n}\right) \leq \hat{u}_{n} \quad \text { for all } n \in \mathbf{N}, \\
\Longrightarrow & \hat{u}^{*} \leq u_{v}^{*} \quad(\text { see }(3.17),(3.24)), \\
\Longrightarrow & \hat{u}^{*}=u_{v}^{*}=\tau(v), \\
\Longrightarrow & \tau(\cdot) \text { is continuous. }
\end{aligned}
$$

From the above arguments it is also clear that, if $E \subseteq C_{+}$is bounded, then

$$
\tau(E) \subseteq C_{+} \text {is relatively compact. }
$$

We conclude that the map $\tau(\cdot)$ is compact.

We introduce the following set

$$
L=\left\{u \in C_{+}: u=t \tau(u), 0<t<1\right\} .
$$

For this set we have the following result.

Proposition 3.4. If hypotheses $\mathrm{H}(\xi), \mathrm{H}(\beta), \mathrm{H}_{0}, \mathrm{H}(\mathrm{f})$ hold, then $L \subseteq C_{+}$is bounded.

Proof. Let $u \in L$. We have

$$
\frac{1}{t} u=\tau(u), \quad 0<t<1 .
$$

Therefore we have

$$
\left\{\begin{array}{l}
-\Delta_{p}\left(\frac{1}{t} u\right)+\xi(z)\left(\frac{1}{t} u\right)^{p-1}=f\left(z, \frac{1}{t} u, D u\right) \quad \text { for a.a. } z \in \Omega \\
\frac{\partial u}{\partial n_{p}}+\beta(z) u^{p-1}=0 \quad \text { on } \partial \Omega .
\end{array}\right.
$$

From (3.25) we obtain

$$
\gamma(u) \leq t^{p-1} \int_{\Omega} f\left(z, \frac{1}{t} u, D u\right) u d z \leq \int_{\Omega} f(z, u, D u) u d z
$$

(see hypothesis $\mathrm{H}(\mathrm{f})(\mathrm{iv})$ and recall that $0<t<1$ ).

On account of hypotheses $\mathrm{H}(\mathrm{f})(\mathrm{i})$, (ii), given $\varepsilon>0$, we can find $c_{13}=c_{13}(\varepsilon)>0$ such that

$$
f(z, x, y) x \leq[\theta(z)+\varepsilon] x^{p}+c_{13} x+\mu|y|^{p-1} x
$$

for a.a. $z \in \Omega$, all $x \geq 0$, all $y \in \mathbf{R}^{N}$.

Using (3.27) in (3.26), via Hölder's inequality, we obtain

$$
\begin{aligned}
& \gamma(u)-\int_{\Omega} \theta(z)|u|^{p} d z-\varepsilon\|u\|^{p}-\frac{\mu}{\hat{\lambda}_{1}^{1 / p}} \gamma(u) \leq c_{14}\|u\| \text { for some } c_{14}>0 \text { (see (2.3)), } \\
\Longrightarrow & \mu_{0} \gamma(u)-\int_{\Omega} \theta(z)|u|^{p} d z-\varepsilon\|u\|^{p} \leq c_{14}\|u\|, \\
\Longrightarrow & {\left.\left[c_{15}-\varepsilon\right]\|u\|^{p-1} \leq c_{14} \quad \text { (see Lemma } 2.5 \text { and hypothesis } \mathrm{H}(\mathrm{f})(\mathrm{ii})\right) . }
\end{aligned}
$$

Choosing $\varepsilon \in\left(0, c_{15}\right)$, we infer that

$$
L \subseteq W^{1, p}(\Omega) \text { is bounded. }
$$

From this as before, using the nonlinear regularity theory ([20, Proposition 2.10] and [15, Theorem 2]), we conclude that $L \subseteq C_{+}$is bounded.

Now we can state the following existence theorem. 
Theorem 3.5. If hypotheses $\mathrm{H}(\xi), \mathrm{H}(\beta), \mathrm{H}_{0}, \mathrm{H}(\mathrm{f})$ hold, then problem (1.1) admits a positive solution $u_{0} \in D_{+}$.

Proof. Propositions 3.3 and 3.4 permit the use of Theorem 2.1 (the LeraySchauder Alternative Theorem). So, we can find $u_{0} \in C_{+}$such that

$$
\begin{aligned}
u_{0} & =\tau\left(u_{0}\right), \\
\Longrightarrow u_{0} & \in S_{u_{0}}^{+} \subseteq D_{+}, \\
\Longrightarrow u_{0} & \in D_{+} \text {is a positive solution of }(1.1) .
\end{aligned}
$$

Acknowledgments. The authors wish to thank the anonymous reviewers for valuable comments and suggestions to improve the presentation. This work was supported by the NSFC (No. 11671111) and Heilongjiang Province Postdoctoral Startup Foundation (Grant No. LBHQ16082).

\section{References}

[1] Aubin, J.-P.: Applied abstract analysis. - Wiley, New York, 1977.

[2] BAI, Y.: Existence of solutions to nonhomogeneous Dirichlet problems with dependence on the gradient. - Electron. J. Differential Equations 2018:101, 2018, 1-18.

[3] Bai, Y, L. Gasinki, and N.S. PApageorgiou: Nonlinear nonhomogeneous Robin problems with dependence on the gradient. - Bound. Value Probl. 2018:17, 2018, 1-24.

[4] Faraci, F., D. Motreanu, and D. Puglisi: Positive solutions of quasi-linear elliptic equations with dependence on the gradient. - Calc. Var. Partial Differential Equations 54, 2015, $525-538$.

[5] Faria, L. F. O., O. H. Miyagaki, D. Motreanu, and M. Tanaka: Existence results for nonlinear elliptic equations with Leray-Lions operator and dependence on the gradient. - Nonlinear Anal. 96, 2014, 154-166.

[6] De Figueiredo, D., M. Girardi, and M. Matzeu: Semilinear elliptic equations with dependence on the gradient via mountain-pass techniques. - Differential Integral Equations 17, 2004, 119-126.

[7] García Melián, J., J. Sabina de Lis, and P. TakÁč: Dirichlet problems for the $p$-Laplacian with a convection term. - Rev. Mat. Complut. 30, 2017, 313-334.

[8] Gasinski, L., and N. S. Papageorgiou: Nonlinear analysis. - Chapman \& Hall/CRC, Boca Raton, Fl, 2006.

[9] Gasinski, L., and N.S. Papageorgiou: Exercises in analysis. Part 1. - Springer, Cham, 2014.

[10] Gasinski, L., and N. S. Papageorgiou: Positive solutions for nonlinear ellitic problems with dependence on the gradient. - J. Differential Equations 263, 2017, 1451-1476.

[11] Gasinski, L., and N. S. Papageorgiou: Positive solutions for the Robin $p$-Laplacian problem with competing nonlinearities. - Adv. Calc. Var. doi.org/10.1515/acv-2016-0039.

[12] Girardi, M., and M. Matzeu: Positive and negative solutions of a quasi-linear elliptic equation by a mountain pass method and truncature techniques. - Nonlinear Anal. 59, 2004, 199210 .

[13] Hu, S., and N.S. Papageorgiou: Handbook of multivalued analysis. Volume I: Theory. Kluwer Academic Publishers, Dordrecht, The Netherlands, 1997.

[14] Huy, N. B., B. T. QuAn, and N. H. Khanh: Existence and multiplicity results for generalized logistic equations. - Nonlinear Anal. 144, 2016, 77-92.

[15] Lieberman, G. M.: Boundary regularity for solutions of degenerate elliptic equations. - Nonlinear Anal. 12, 1988, 1203-1219. 
[16] Motreanu, D., C. Vetro, and F. Vetro: A parametric Dirichlet problem for systems of quasilinear elliptic equations with gradient dependence. - Numer. Funct. Anal. Optim. 37, 2016, $1551-1561$.

[17] Motreanu, D., C. Vetro, and F. Vetro: Systems of quasilinear elliptic equations with dependence on the gradient via subsolution-supersolution method. - Discrete Contin. Dyn. Syst. Ser. S 11, 2018, 309-321.

[18] Mugnai, D., and N. S. Papageorgiou: Resonant nonlinear Neumann problems with indefinite weight. - Ann. Sc. Norm. Super. Pisa Cl. Sci. (5) 11, 2012, 729-788.

[19] Papageorgiou, N.S., and V. D. Radulescu: Multiple solutions with precise sign for parametric Robin problems. - J. Differential Equations 256, 2014, 1449-2479.

[20] Papageorgiou, N. S., and V. D. Radulescu: Nonlinear nonhomogeneous Robin problems with superlinear reaction term. - Adv. Nonlinear Stud. 16, 2016, 737-764.

[21] Papageorgiou, N. S., V. D. Radulescu, and D. D. Repovs: Positive solutions for perturbations of the Robin eigenvalue problem plus an indefinite potential. - Discrete Contin. Dyn. Syst. 37, 2017, 2589-2618.

[22] Papageorgiou, N.S., V.D. Radulescu, and D. D. Repovs: Resonant Robin problems driven by the $p$-Laplacian plus an indefinite potential. - Ann. Acad. Sci. Fenn. Math. 43, 2018, 483-508.

[23] Papageorgiou, N. S., V. D. Radulescu, and D. D. Repovs: Positive solutions for nonlinear nonhomogeous parametric Robin problems. - Forum Math. 30, 2018, 553-580.

[24] Papageorgiou, N. S., V. D. Radulescu, and D. D. Repovs: Nonlinear elliptic inclusions with unilateral constraint and dependence on the gradient. - Appl. Math. Optim. 78, 2018, $1-23$.

[25] Papageorgiou, N. S., V. D. Radulescu, and D. D. Repovs: Positive solutions for nonvariational Robin problems. - Asymptot. Anal. 108, 2018, 243-255.

[26] Ruiz, D.: A priori estiamtes and existence of positive solutions for strongly nonlinear problems. - J. Differential Equations 199, 2004, 96-114.

[27] TANAKA, M.: Existence of a positive solution for quasilinear elliptic equations with nonlinearity including the gradient. - Bound. Value Probl. 2013:173, 2013, 1-11.

Received 3 October 2018 • Accepted 29 November 2018 